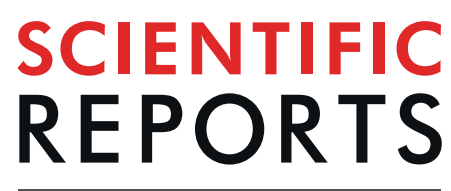

natureresearch

\title{
OPEN MicroRNA-509-3p inhibits cellular migration, invasion, and proliferation, and sensitizes osteosarcoma to cisplatin
}

\author{
Sagar L. Patil $\mathbb{1}^{1}$, Asha Palat ${ }^{2}$, Yinghong Pan ${ }^{2}$, Kimal Rajapakshe ${ }^{3}$, Rachna Mirchandani ${ }^{2}$, \\ Maria Bondesson ${ }^{4}$, Jason T. Yustein ${ }^{5}$, Cristian Coarfa $\mathbb{1}^{3}$ \& Preethi H. Gunaratne ${ }^{2 *}$
}

Osteosarcoma (OS) is the most common primary pediatric malignancy of the bone having poor prognosis and long-term survival rates of less than $30 \%$ in patients with metastasis. MicroRNA-509 was reported to be downregulated in OS. We and others previously published that miR-509-3p can strongly attenuate cellular migration/invasion and sensitize ovarian cancer to cisplatin. Here, we show that overexpression of miR-509-3p inhibited migration of primary OS cell lines U2OS, HOS, and SaOS2 as well as metastatic derivatives 143B and LM7. miR-509-3p overexpression also inhibited proliferation and invasion of $\mathrm{HOS}$ and 143B cells and sensitized cells to cisplatin. Luciferase reporter assays using 3'-UTRs of predicted miR-509-3p targets associated with metastatic phenotypes revealed ARHGAP1 could be one of the downstream effectors of miR-509-3p in HOS. To find the global impact of miR509-3p overexpression and cisplatin treatment we performed Reverse Phase Protein Analysis (RPPA). $A X L$, which has been reported to play a critical role in cisplatin resistance and confirmed as direct target of miR-509-3p was downregulated upon miR-509-3p treatment and further down-regulated upon miR509-3p + cisplatin treatment. We propose that the miR-509-3p/AXL and miR-509-3p/ARHGAP1 axes have the potential to uncover new druggable targets for the treatment of drug resistant metastatic osteosarcoma.

Osteosarcoma (OS), which arises primarily in children and adolescents, is the most common primary malignancy of the bone and the most frequent cause of cancer-related death in children ${ }^{1,2}$. Currently, combination chemotherapy consisting of doxorubicin, cisplatin and methotrexate, along with surgery is used to treat $\mathrm{OS}^{1}$. The 5 -year survival is nearly $75 \%$ for patients with no evidence of metastatic disease at diagnosis in contrast to $30 \%$ for patients who present with gross evidence of metastatic lesions on radiographic imaging ${ }^{3}$. Approximately $20 \%$ of the OS patients present with metastases and $\sim 50 \%$ of patients who experience OS recurrence develop metastasis within 18 months after chemotherapy ${ }^{3}$. Pulmonary metastasis is the most common site and most common cause of death of OS patients ${ }^{3,4}$. Systemic chemotherapy has increased long-term survival rates up to nearly $75 \%$ in patients with localized OS, but has had a minimal impact on overall survival of patients with metastatic disease ${ }^{5,6}$. New approaches targeting metastasis-specific cellular pathways therefore have been a major focus ${ }^{7}$. However, unlike cancers with reciprocal chromosomal translocations, OS has complex karyotypes with multiple genetic alterations ${ }^{8}$. Whole-genome sequencing of tumors from 32 OS patients showed that cancer-specific TP53 rearrangements were found in more than $50 \%$ of patients 9 .

MicroRNAs are small $\sim 22$ nucleotide long endogenous non-coding RNAs that have important regulatory roles in post-transcriptional gene silencing of hundreds of targets within and between diverse cellular pathways ${ }^{10}$. microRNA-509 (miR-509-5p), was reported to be downregulated, in OS tissues and cell lines ${ }^{11}$. This observation also suggests that miR-509-3p, which is transcribed along with miR-509-5p, is downregulated in OS tissues. Previously, we reported that miR-509-3p is a strong tumor suppressor that attenuates migration and disrupts

${ }^{1}$ Department of Pediatrics, Columbia University, New York, NY, USA. ${ }^{2}$ Department of Biology and Biochemistry, University of Houston, Houston, TX, USA. ${ }^{3}$ Department of Molecular and Cellular Biology, Baylor College of Medicine, Houston, TX, USA. ${ }^{2}$ Department of Intelligent Systems Engineering, Indiana University, Bloomington, IN, USA. ${ }^{5}$ Texas Children's Cancer and Hematology Centers, Department of Pediatrics, Baylor College of Medicine, Houston, TX, USA. *email: phgunaratne@uh.edu 
A
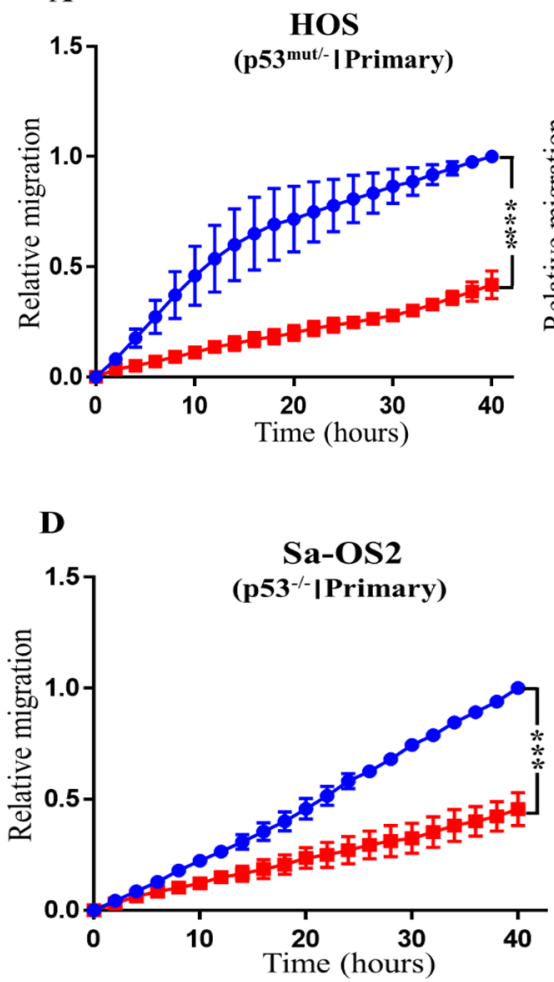

B

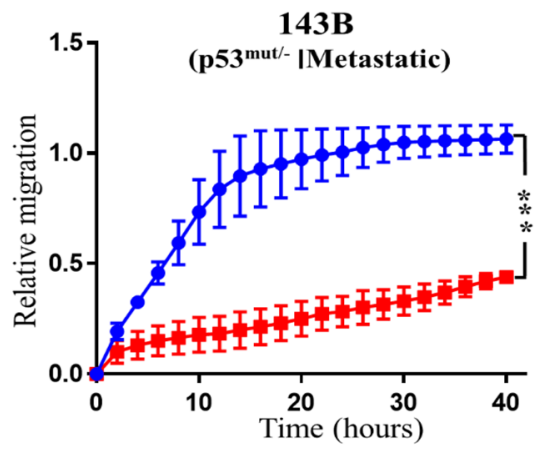

E

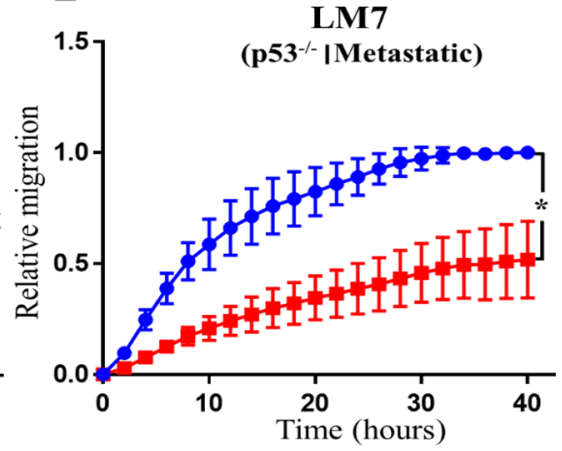

C

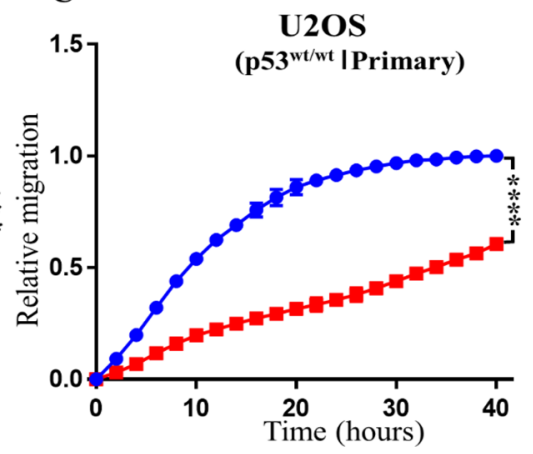

Figure 1. miR-509-3p inhibits OS migration. In vitro 96 well plate scratch/wound healing assay after transiently overexpressing miR-509-3p mimic and scrambled negative control RNA (NC) in (A) HOS (p53 ${ }^{\text {mut/- }}$, primary) and its metastatic derivative (B) 143B (p53 $3^{\mathrm{mut} /-}$ ); (C) U2OS (p53 ${ }^{\mathrm{wt} / \mathrm{wt}}$, primary); (D) Sa-OS2 (p53 ${ }^{-/-}$, primary) and it's metastatic derivative (E) LM7 (p53 $\left.{ }^{-1-}\right)$ cell lines. $(\mathrm{N} \geq 3)$. Migration at all time points was normalized to migration at the final time point ( 40 hours) of cells treated with NC to obtain relative migration. Error bars represent standard errors of mean (SEM) between biological replicates. Time course starts $\sim 72 \mathrm{~h}$ post transfection. P values were calculated at $24 \mathrm{~h}$ intervals between NC and miR509-3p treated cells $(* \mathrm{p} \leq 0.05$, $* * \mathrm{p} \leq 0.01),(* * * \mathrm{p} \leq 0.001, * * * * \mathrm{p} \leq 0.0001)$.

multi-cellular spheroids in multiple ovarian cancer (OVCA) cell lines ${ }^{12}$. In the p53-mutant ovarian cancer line OVCAR8, we established YAP1 to be a direct downstream target and a critical effector of miR-509-3p-mediated tumor suppression ${ }^{12}$. YAP1 is highly expressed in both human and mouse OS and knockdown of YAP1 was found to reduce OS tumor progression in mouse models ${ }^{13}$. We therefore, hypothesized that the miR-509-3p/YAP1 axis can be used to identify and develop new druggable targets for metastatic OS.

In this study, we investigated the role of miR-509-3p in migration and invasion of primary and metastatic OS cell lines. Cisplatin is one of the most commonly used drugs for the treatment of OS ${ }^{14,15}$. Cisplatin has been reported to inhibit migration and invasion of ovarian cancer ${ }^{16}$. We examined the role of miR-509-3p in migration and invasion of OS cells in the presence or absence of the chemotherapeutic drug cisplatin and through microRNA target predictions and Reverse Phase Protein Array (RPPA) we identified downstream effectors of miR-509-3p.

Our study found that miR-509-3p overexpression inhibited OS migration and invasion and sensitized OS to cisplatin. Downregulation of direct targets ARHGAP1 and AXL in miR-509-3p treated OS cell lines and further downregulation of AXL upon miR-509-3p + cisplatin treatment suggests that the miR-509-3p/AXL axis could reveal genes and pathways that drive cisplatin resistance in OS.

\section{Results}

miR-509-3p inhibits OS migration. To assess ability of miR-509-3p to inhibit OS migration we performed 96 well plate scratch/wound healing assays on five OS cell lines including primary HOS (p53 $3^{\mathrm{mut} /-}$ ) and its metastatic derivative 143B ( $\left.\mathrm{p} 53^{\mathrm{mut} /-}\right)$; primary SaOS2 $\left(\mathrm{p} 53^{-/-}\right)$and it metastatic derivative LM7 ( $\mathrm{p} 53^{-/-}$); and primary $\mathrm{U} 2 \mathrm{OS}\left(\mathrm{p} 53^{\mathrm{wt} / \mathrm{wt}}\right.$ ) cell line (Fig. 1). Transient overexpression of miR-509-3p mimc strongly inhibited migration in all five cell lines as compared to a negative control scrambled RNA (NC) transfected cells. At the $24 \mathrm{~h}$ time point, the relative migration of HOS transfected with miR-509-3p mimc was 0.23 units (arbitrary units relative to NC at the final time point) compared to 0.77 units in $\mathrm{NC}(\mathrm{P}=0.004)$ (Fig. 1A). The relative migration of $143 \mathrm{~B}\left(\mathrm{p} 53^{\mathrm{mut} /-}\right)$ cells transfected with miR-509-3p mimc was 0.28 units compared to 1.00 units in NC treatment $(\mathrm{P}=0.004)$ (Fig. 1B). The relative migration of U2OS (p53 ${ }^{\text {wt/wt }}$ ) cells transfected with miR-509-3p mimc was 0.35 units as compared to 0.91 units in NC $\left(\mathrm{P}=9.39 \mathrm{e}^{-005}\right)$ (Fig. 1C). Primary SaOS2 $\left(\mathrm{P}^{2} 3^{-1-}\right)$ cells with null p53 (slow growing and less metastatic), migrated slower than the other four cell lines, and miR-509-3p overexpression further 
A

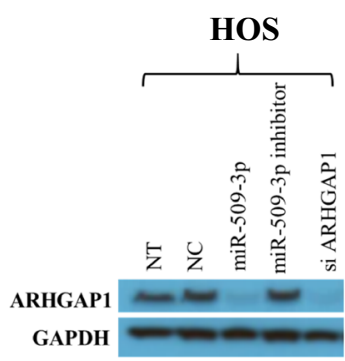

C

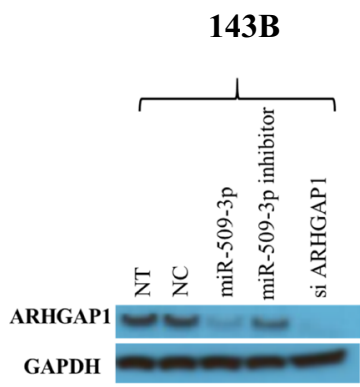

B

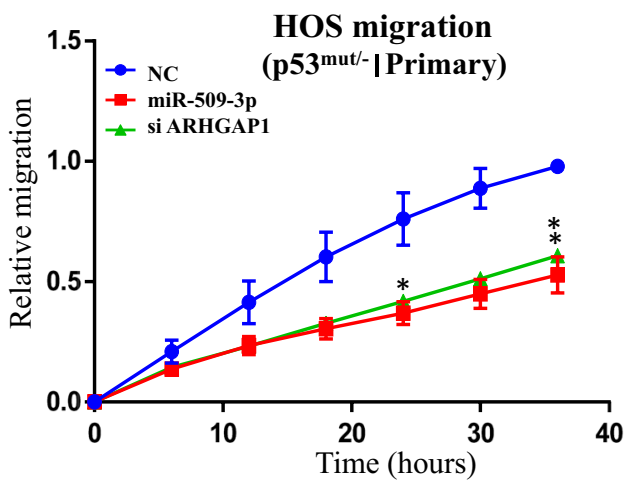

D

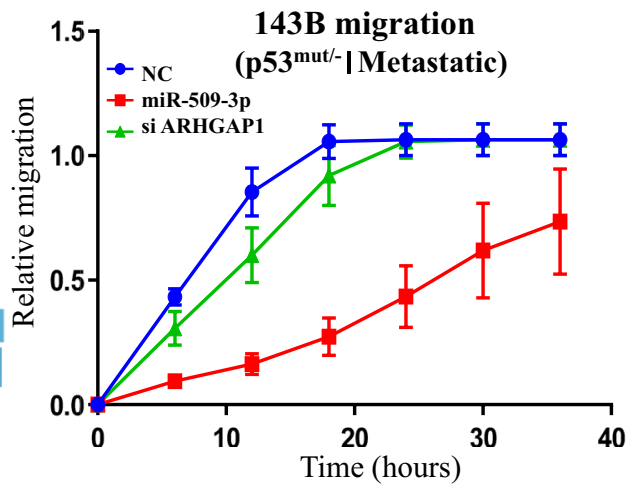

$\mathbf{E}$

Predicted binding site of miR-509-3p on ARHGAP1 3'UTR

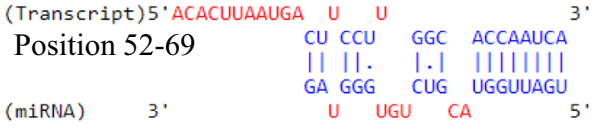

miR-509-3p targets ARHGAP1 3'UTR

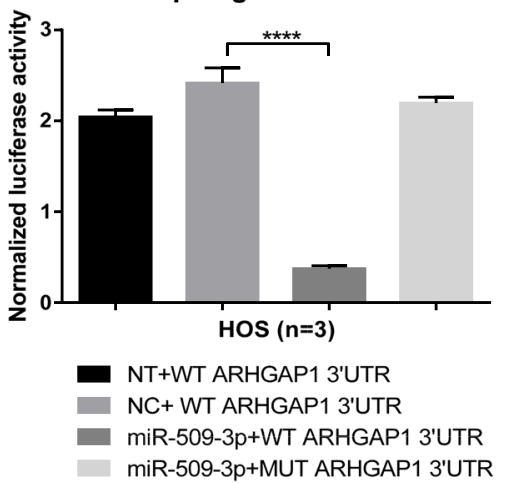

Figure 2. ARHGAP1 is a critical effector of miR-509-3p-mediated migration inhibition. (A) Western blot analysis of ARHGAP1 protein level in HOS cells, $72 \mathrm{~h}$ post transfection of miR-509-3p mimic and NC. (B) In vitro 96 well plate scratch plate/wound healing assay in HOS cells. (C) Western blot analysis of ARHGAP1 protein level in 143B cells, $72 \mathrm{~h}$ post transfection of miR-509-3p mimic and NC. Blots presented here are cropped from same blot and full-length blots are presented in Supplementary Fig. 3. (D) In vitro 96 well plate scratch plate/wound healing assay in 143B cells and (E) TargetScan prediction of miR-509-3p binding site on $3^{\prime}$ UTR of ARHGAP1 and ARHGAP1 luciferase reporter activity for the predicted binding site, $48 \mathrm{~h}$ post transfection of miR-509-3p mimic and NC into HOS cells.

inhibited its migration. The relative migration of SaOS2 cells transfected with miR-509-3p mimc was 0.27 units compared to 0.58 units in NC $(\mathrm{P}=0.004)$ (Fig. 1D). The relative migration of LM7 $\left(\mathrm{P}^{2} 3^{-1-}\right)$ cells transfected with miR-509-3p mimc was 0.38 units compared to 0.89 units in $\mathrm{NC}(\mathrm{P}=0.011)$ (Fig. 1E). Collectively these results demonstrate that miR-509-3p inhibits OS cell migration irrespective of p53 status or metastatic capability of OS.

ARHGAP1 is a direct downstream target of miR-509-3p and a critical effector of miR-5093p-mediated migration inhibition. To elucidate downstream effectors of miR-509-3p-driven attenuation of migration in OS cells, we used siRNAs against genes that were predicted targets of miR-509-3p, and were associated with ECM and cell adhesion (Supplementary Fig. 1A). In our previous work, we established that YAP1 is a direct downstream target and a critical effector of miR-509-3p in the p53-mutated ovarian cancer cell line OVCAR $8^{12}$. In contrast to OVCAR8 cells, we found that siRNA to YAP1 had little effect on HOS and 143B cell migration (Supplementary Fig. 1A). By sharp contrast, knockdown of another predicted target gene, ARHGAP1, by siRNA inhibited HOS cell migration to the same extent as miR-509-3p mimic (Fig. 2B and supplementary Fig. 1A). The relative migration of HOS cells transfected with siARHGAP1 was 0.41 units $(\mathrm{P}=0.034)$ and the relative migration of HOS cells overexpressing miR-509-3p was 0.37 units $(\mathrm{P}=0.030)$ compared to 0.76 units in $\mathrm{NC}$ at $24 \mathrm{~h}$ (Fig. 2B).

Overexpression of miR-509-3p in OS cell lines resulted in downregulation of mRNA and protein levels of ARHGAP1 (Fig. 2A and Supplementary Fig. 1C,D). To confirm actual binding of miR-509-3p to its predicted binding site on 3'-UTR of ARHGAP1 gene (Fig. 2E), we performed a luciferase reporter assay in HOS cells. Luciferase signal was significantly repressed and this repression was lost in HOS cells carrying a luciferase reporter with mutations in the $3^{\prime}$-UTR of ARHGAP1 (Fig. 2E). This confirms that ARHGAP1 is a direct target of miR-509-3p.

Interestingly, siARHGAP1 had no impact on migration of the metastatic 143B cell line even though Western blots confirmed that overexpression of miR-509-3p led to down-regulation of ARHGAP1 protein in both HOS 

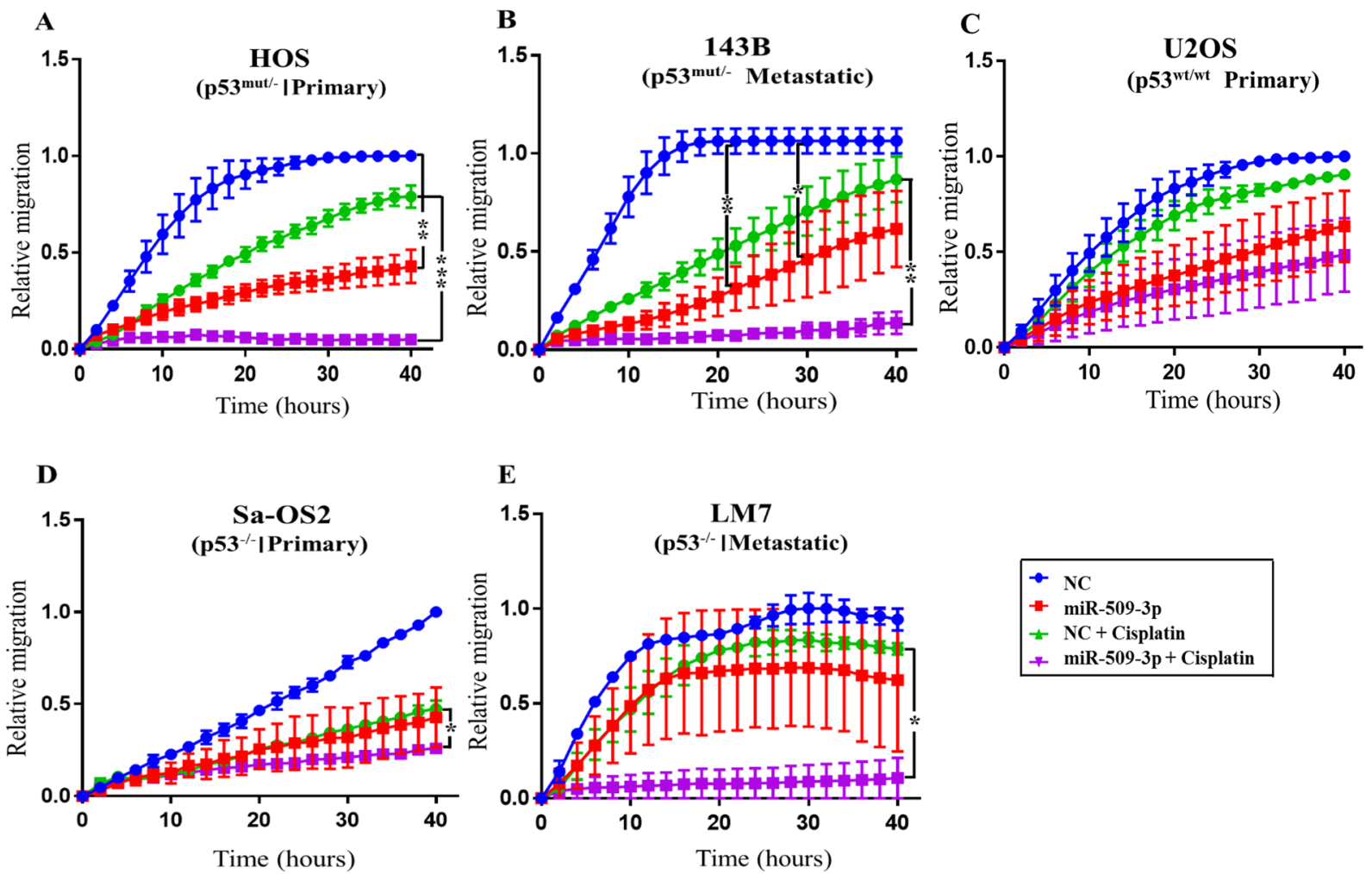

Figure 3. Cellular migration assays performed on 5 OS cell lines treated with miR-509-3p mimic + cisplatin. In vitro 96 well plate scratch plate/wound healing assay were performed after transiently overexpressing miR509-3p mimic and NC with and with and without cisplatin $(2.5 \mathrm{ug} / \mathrm{ml})$. The cell lines tested include (A) HOS (p53 ${ }^{\mathrm{mut} /-}$, Primary) and its metastatic derivative (B) 143B (p53 ${ }^{\mathrm{mut} /-}$ ); (C) U2OS (p53 ${ }^{\mathrm{wt} / \mathrm{wt}}$, Primary); (D) SaOS2 (p53 $3^{-l-}$, Primary) and its metastatic derivative (E) LM7 ( $\left.553^{-l-}\right)$ cell line. $(\mathrm{N} \geq 3$ except LM7 N=2). Migration at all time points was normalized to migration at final time point $(40 \mathrm{~h})$ of cells treated with $\mathrm{NC}$ to get relative migration. Error bars represent SEM between biological replicates. Time course starts $\sim 72 \mathrm{~h}$ post transfection. P values are calculated at 24 hour between NC and miR-509-3p and between NC + cisplatin and miR-509-3p + cisplatin treated cells $(* \mathrm{p} \leq 0.05, * * \mathrm{p} \leq 0.01),(* * * \mathrm{p} \leq 0.001, * * * * \mathrm{p} \leq 0.0001)$.

and 143B cells, which had almost equal amounts of ARHGAP1 (Fig. 2A-D). Relative migration of 143B cells treated with siARHGAP1 was 1.00 units and relative migration of cells treated with miR-509-3p was 0.43 units compared to 1.00 units in $\mathrm{NC}$ at $24 \mathrm{~h}$ (Fig. 2D).

miR-509-3p inhibits invasion and proliferation of HOS and 143B cell lines and sensitizes cells to cisplatin. Cisplatin is one of the most commonly used drugs for the treatment of OS ${ }^{14,15}$. Cisplatin has been reported to inhibit migration and invasion of ovarian cancer ${ }^{16}$. To test the impact of the combination of miR-509-3p and cisplatin, we performed 96-well plate scratch/wound healing assay with all five OS cell lines transfected with miR-509-3p in combination with cisplatin treatment $(2.5 \mu \mathrm{g} / \mathrm{ml})$ (Fig. 3). Combination of miR$509-3 p$ and cisplatin treatment (miR-509-3p + cisplatin) completely inhibited migration of the primary cell line HOS (p53 ${ }^{\mathrm{mut} /-}$ ) and its metastatic derivative 143B (p53 ${ }^{\mathrm{mut} /-}$ ), and LM7 (p53 ${ }^{-/-}$), a metastatic derivative of SaOS2 compared NC, miR-509-3p alone or NC with cisplatin (NC+ cisplatin) (Fig. 3A,B,E). In HOS cells relative migration was 0.04 units in miR-509-3p + cisplatin treatment, 0.33 units in miR-509-3p treatment and 0.56 units in $\mathrm{NC}+$ cisplatin treatment compared to 0.94 units in $\mathrm{NC}$ at $24 \mathrm{~h}$. In the $143 \mathrm{~B}$ cell line, relative migration was 0.08 units in miR-509-3p + cisplatin treatments, 0.34 units in miR-509-3p treatment and 0.57 units in NC + cisplatin treatments compared to 1.06 units in NC at $24 \mathrm{~h}$. In the LM7 cell line, relative migration was 0.08 units in miR$509-3 p+$ cisplatin treatments, 0.68 units in miR-509-3p treatments and 0.82 units in $\mathrm{NC}+$ cisplatin treatments compared to 0.92 units in NC at $24 \mathrm{~h}$. Combination of miR-509-3p and cisplatin treatment had little effect on cell migration of primary cell line U2OS (p53 $\left.{ }^{\mathrm{wt} / \mathrm{wt}}\right)$ and Sa-OS2 (p53 ${ }^{-/-}$) compared to cells treated with miR-509-3p alone (Fig. 3C,D). In U2OS cell line, relative migration was 0.34 units in miR-509-3p + cisplatin treatments, 0.42 units in miR-509-3p treatment and 0.76 units is in $\mathrm{NC}+$ cisplatin treatments compared to 0.90 units in NC at $24 \mathrm{~h}$. In the Sa-OS2 cell line, relative migration was 0.18 units in miR-509-3p + cisplatin treatments, 0.29 units in miR-509-3p treatment and 0.29 units in $\mathrm{NC}+$ cisplatin treatments compared to 0.56 units in $\mathrm{NC}$ at $24 \mathrm{~h}$. We conclude that a combination of miR-509-3p transfection and cisplatin treatment reduced cell migration more efficiently than either compound alone in OS cell lines, although to a varying degree in the different cell lines.

In order to study the effect of miR-509-3p on other cancer related phenotypes in OS, we focused on the primary HOS cell line and its metastatic derivative 143B. To test the impact of miR-509-3p on invasion and 
Invasion

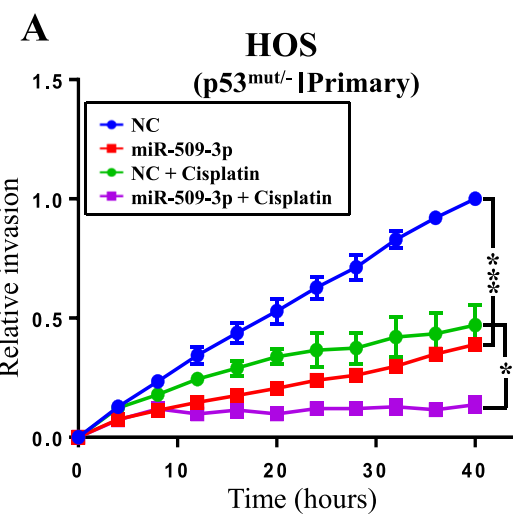

C

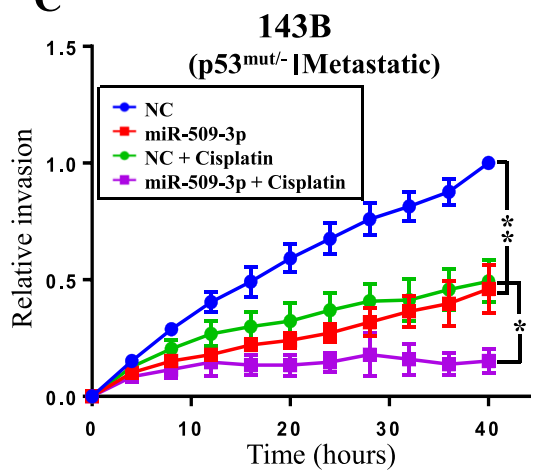

Proliferation

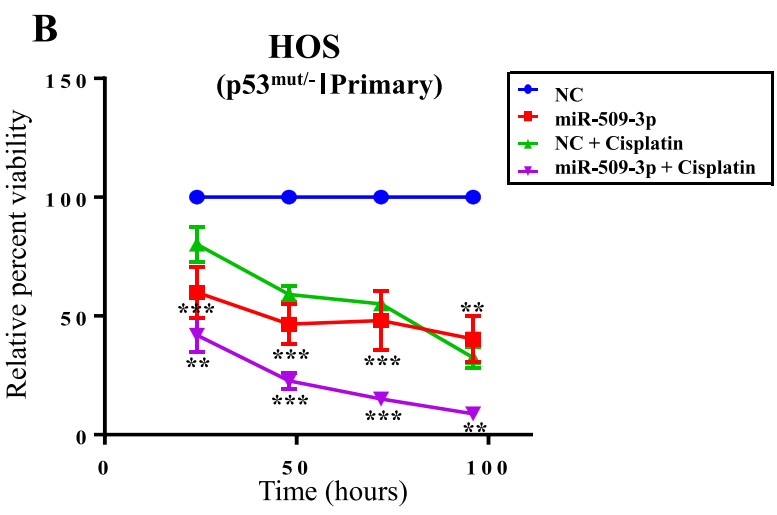

D

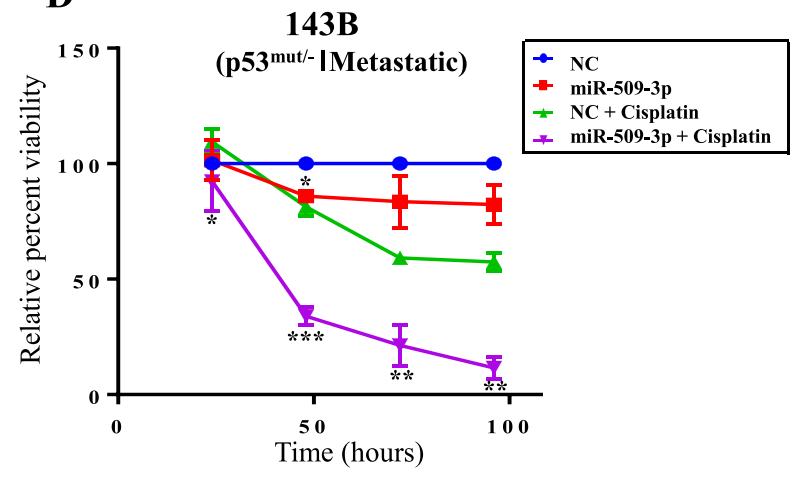

Figure 4. miR-509-3p inhibits OS invasion and proliferation, and sensitizes OS to cisplatin. In vitro 96 well plate Matrigel invasion assay after transiently overexpressing miR-509-3p mimic and NC with and with and without cisplatin $(2.5 \mathrm{ug} / \mathrm{ml})$ in $(\mathbf{A})$ HOS and $(\mathbf{C}) 143 \mathrm{~B}$ cells. $(\mathrm{N}=3)$. Invasion at all time points was normalized to invasion at final time point $(40 \mathrm{~h})$ of cells treated with $\mathrm{NC}$ to get relative invasion. Error bars represent SEM between biological replicates. Time course starts $\sim 72 \mathrm{~h}$ post transfection. Cells proliferation assay (MTS assay) after transiently overexpressing miR-509-3p mimic and $\mathrm{NC}$ with and without cisplatin $(2.5 \mathrm{ug} / \mathrm{ml})$ in B) HOS and D) 143B cells. $(\mathrm{N}=3)$. P values are calculated at 24, 48, 72 and $96 \mathrm{~h}$ between NC and miR-509-3p and between NC + cisplatin and miR-509-3p + cisplatin treated cells $(* p \leq 0.05, * * p \leq 0.01),(* * * p \leq 0.001$, $* * * * \mathrm{p} \leq 0.0001)$.

proliferation of HOS and 143B cells, we performed Matrigel invasion and proliferation assays (Fig. 4). miR509-3p strongly inhibited invasion and proliferation of HOS cells compared to NC (Fig. 4A,B). The combination of miR-509-3p and cisplatin $(2.5 \mu \mathrm{g} / \mathrm{ml})$ had an even greater impact on inhibiting invasion and proliferation of HOS cells (Fig. 4A,B). In HOS cells the relative invasion was 0.12 units in miR-509-3p + cisplatin treatments, 0.23 units in miR-509-3p treatment and 0.36 units in $\mathrm{NC}+$ cisplatin treatments compared to 0.62 units in $\mathrm{NC}$ at $24 \mathrm{~h}$ (Fig. 4A). In HOS cells relative percent viability was $8.75 \%$ in miR-509-3p + cisplatin treatments, $40.32 \%$ in miR509-3p treatment and $32.43 \%$ in NC + cisplatin treatments compared to $100 \%$ in NC treated cells at $96 \mathrm{~h}$ (Fig. 4B).

miR-509-3p significantly inhibited invasion in 143B cells, but had only a moderate effect on proliferation compared to NC (Fig. 4C,D). The combination of miR-509-3p + cisplatin $(2.5 \mu \mathrm{g} / \mathrm{ml})$ had the largest impact on inhibiting invasion and proliferation of $143 \mathrm{~B}$ cells (Fig. 4C,D). In $143 \mathrm{~B}$ cells, the relative invasion was 0.14 units in miR-509-3p + cisplatin treatments, 0.27 units in miR-509-3p treatment and 0.37 units in NC + cisplatin treatments compared to 0.67 units in NC at $24 \mathrm{~h}$. In $143 \mathrm{~B}$ cells, relative percent viability was $11.50 \%$ in miR509-3p + cisplatin treatments, $57.45 \%$ in miR-509-3p treatment and $82.32 \%$ in NC + cisplatin treatments compared to $100 \%$ in $\mathrm{NC}$ at $96 \mathrm{~h}$.

RPPA revealed AXL down-regulation as one of the modes of miR-509-3p mediated cisplatin sensitivity. To uncover downstream effectors of miR-509-3p action in HOS and 143B cells, we performed reverse phase protein array, in which the expression levels of 296 different proteins involved in cancer development and progression were measured after miR-509-3p mimic or control transfections. We observed that in HOS and 143B cells, overexpression of miR-509-3p mimic resulted in a reduced AXL protein expression compared to NC (Fig. 5A,B). Earlier reports have shown that downregulation of AXL inhibits 143B migration and invasion $^{8,17}$. Western blot analysis showed that the AXL protein was downregulated in miR-509-3p treated 143B cells (Fig. 5C). We confirmed AXL to be a direct target of miR-509-3p by luciferase reporter assay (Fig. 5D). To uncover critical downstream effectors of miR-509-3p + cisplatin combination, we compared protein expression changes between miR-509-3p + cisplatin and NC + cisplatin treated cells. We observed that AXL, which is known to drive acquired resistance to cisplatin, were downregulated in both HOS and 143B cell lines treated with 
A

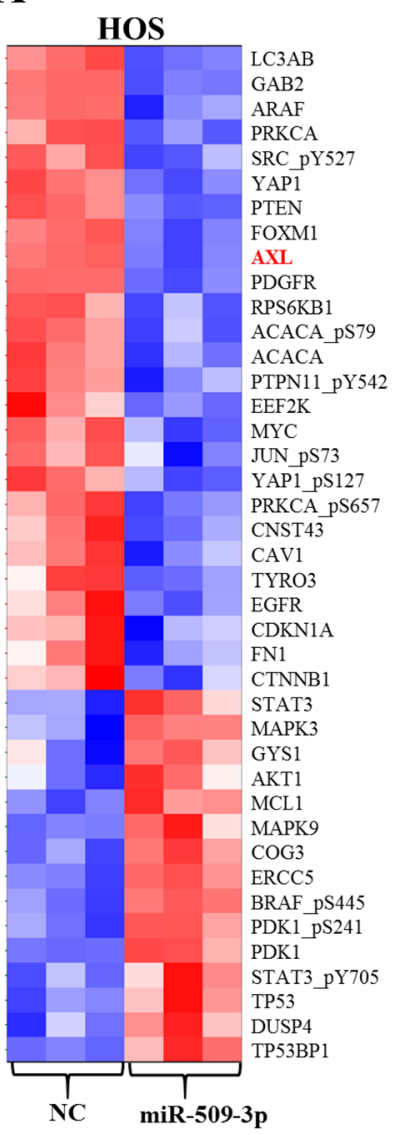

B

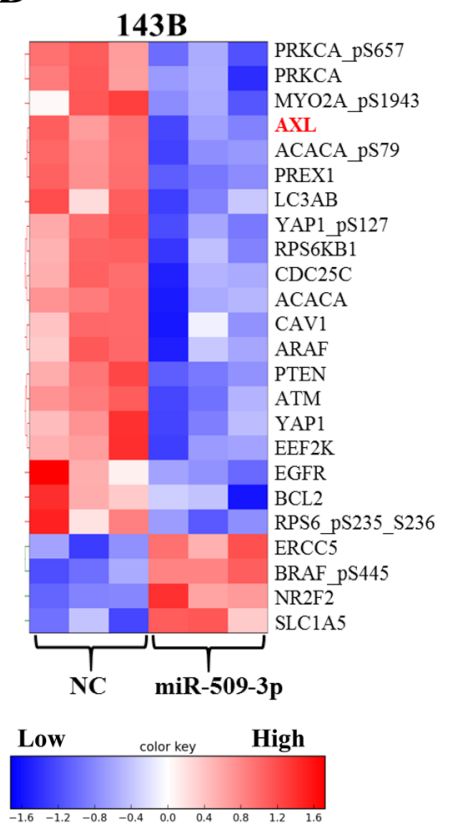

Predicted binding site of miR-509-3p on AXL 3'UTR

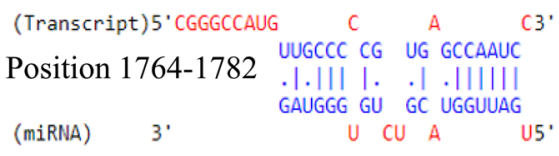

C

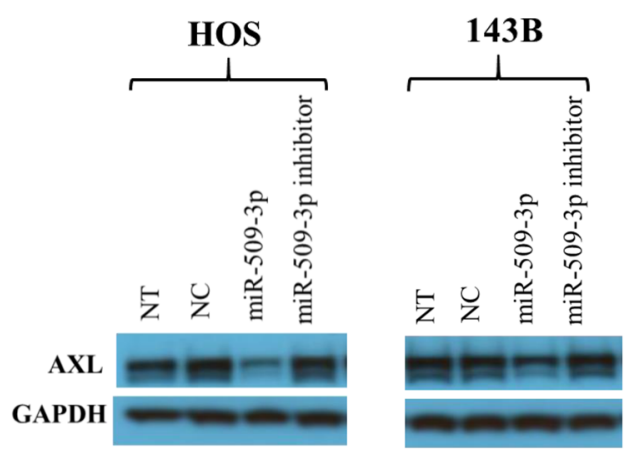

miR -509-3p targets AXL 3'UTR

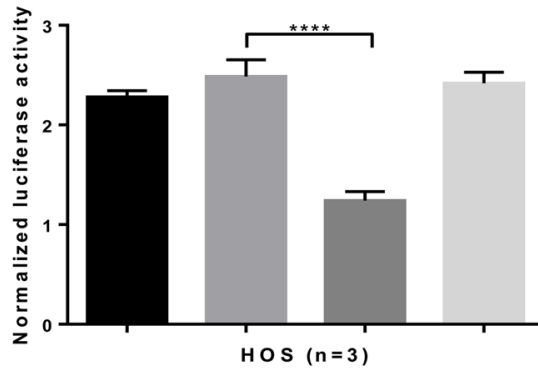

NT+ WT AXL 3'UTR
NC+ WT AXL 3'UTR
miR-509-3p+ WT AXL 3'UTR
miR-509-3p+ MUT AXL 3'UTR

Figure 5. miR-509-3p downregulates AXL expression. Heat map depiction of proteins differentially expressed between NC and miR-509-3p overexpression in (A) HOS and (B) 143B cells. Protein expression was assessed by reverse phase protein array (RPPA). (C) Western blot analysis of AXL protein level, $72 \mathrm{~h}$ post transfection in HOS and 143B cells. Blots presented here are cropped from same blot and full-length blots are presented in Supplementary Fig. 3. (D) TargetScan prediction of miR-509-3p binding site on $3^{\prime} \mathrm{UTR}$ of AXL and AXL luciferase reporter activity for the predicted binding site, $48 \mathrm{~h}$ post transfection of miR-509-3p mimic and NC into HOS cells.

miR-509-3p + cisplatin compared to NC + cisplatin treatments (Fig. 6A-D) ${ }^{18-21}$. The combination of siAXL and cisplatin decreased proliferation to $42.6 \%$ (Fig. 6E). In addition to AXL, other genes such as ATM involved in cisplatin resistance and which are not direct target of miR-509-3p were also downregulated in miR-509-3p + cisplatin treatment. This suggests that miR-509-3p sensitizes OS cells to cisplatin partly by directly downregulating AXL and partly by indirectly downregulating other genes involved in cisplatin resistance.

\section{Discussion}

miR-509-3p, expressed from a $\sim 100 \mathrm{~kb}$ genomic cluster of miRNAs on Xq27.3, is emerging as a strong tumor suppressor in relation to multiple cancer types. Zhang et al. 2017, reported that miR-509-5p is downregulated in OS which suggests that miR-509-3p, which is expressed on the same hairpin as miR-509-5p is also downregulated in OS. miR-509-3p has been established to inhibit proliferation and increase sensitivity to cisplatin in chemo-resistant ovarian cancer cells and influence the cell cycle, colony formation and migration of human epithelial lung and cervical cancer cell line $\mathrm{e}^{12,22,23}$. Here we report that miR-509-3p is also a strong tumor suppressor of OS, in which it inhibits cellular migration, invasion and proliferation and also sensitizes OS cells to cisplatin. Although previous work from our laboratory, established YAP1 to be a direct downstream target and a critical effector of miR-509-3p-driven inhibition of migration in OVCAR8 ${ }^{12}$, we found that this is not the case for OS cells. siRNA to YAP1 had no effect on HOS and 143B cell migration (Supplementary Fig. 1A,B). This is likely because microRNAs act through different genetic networks in cells of different genetic backgrounds. Instead, we found that ARHGAP1 (CDC42 GTPase-Activating Protein1) which is also associated with ECM and cell adhesion to be a direct downstream target and critical effector of miR-509-3p-mediated inhibition of migration in HOS cells. ARHGAP1 is also a direct target of miR-34a and downregulation of ARHGAP1 alone was sufficient to inhibit human lung adenocarcinoma invasion ${ }^{24}$. Interestingly, we found that although miR-509-3p was able to downregulate ARHGAP1 expression in both HOS and 143B cells the ability of siARHGAP1 to inhibit migration and invasion was limited to HOS cells. HOS and 143B are derived from the same patient however 143B has Ras 
A

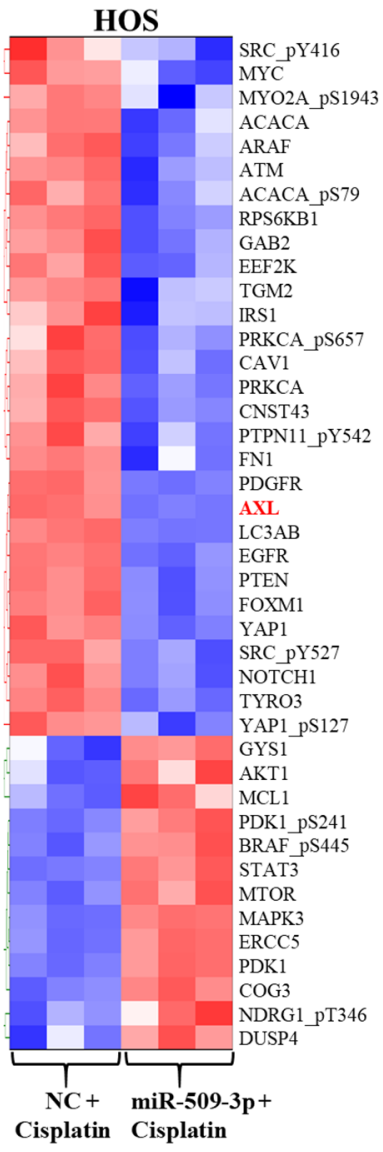

B
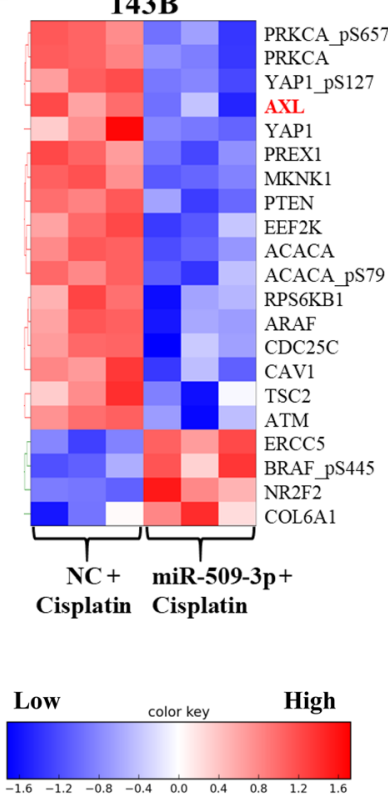

C

HOS

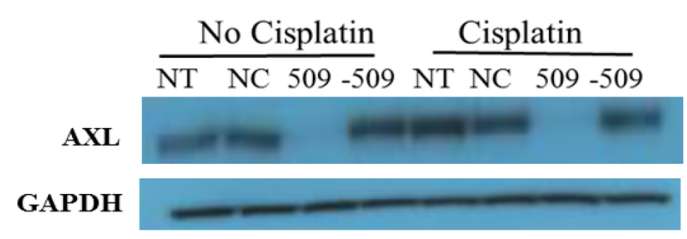

143B

D

$\frac{\text { No Cisplatin }}{\text { NT NC } 509-509} \frac{\text { Cisplatin }}{\text { NT NC } 509-509}$

AXL

GAPDH

E

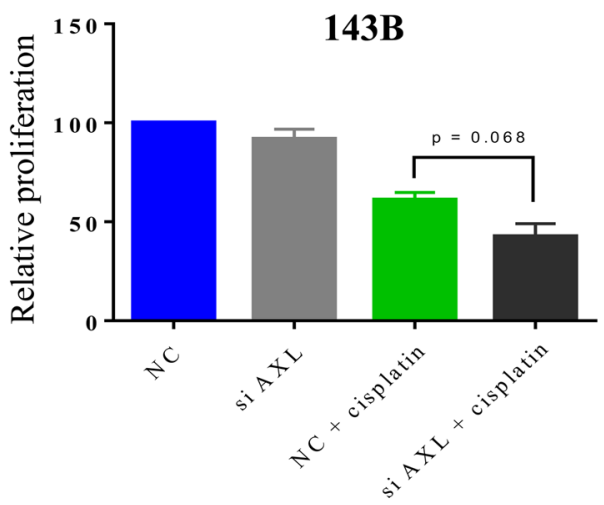

Figure 6. RPPA analysis on HOS and 143B treated with miR-509-3p + cisplatin, and effect of siAXL + cisplatin on 143B. Heat map depiction of proteins differentially expressed between NC and miR-509-3p overexpression in (A) HOS and (B) 143B cells with and without cisplatin treatment. Western blot analysis of AXL protein level, $72 \mathrm{~h}$ post transfection of NT, NC, miR-509-3p (509), and miR-509-3p inhibitor (-509) in (C) HOS and (D) 143B cells with and without cisplatin treatment. Blots presented here are cropped from same blot and full-length blots are presented in Supplementary Fig. 4. (E) Proliferation assay (MTS assay) in 143B cells transfected with $\mathrm{NC}$ and siAXL with and without cisplatin at $72 \mathrm{~h}$ post transfection. $(\mathrm{N}=3$, except $\mathrm{E}, \mathrm{N}=2)$. Error bars represent SEM between biological replicates. P values calculated between $\mathrm{NC}+$ cisplatin and siAXL + cisplatin treated cells $(* \mathrm{p} \leq 0.05, * * \mathrm{p} \leq 0.01),(* * * \mathrm{p} \leq 0.001, * * * * \mathrm{p} \leq 0.0001)$.

stable expression. We propose that this and other mutations that may have accumulated during metastatic progression of HOS may have allowed 143B cells to circumvent the dependence on ARHGAP1 and utilize alternate pathways for driving cellular migration. This observation adds to the reports from us and other groups that, microRNAs can have different phenotypic impacts in different cell types even within same tumor type.

In order to uncover downstream effectors of miR-509-3p in the metastatic derivative $143 \mathrm{~B}$ cell line, we performed RPPA and observed that AXL was among the genes that were significantly downregulated following miR-509-3p treatment of both HOS and 143B cells. Knockdown of AXL in 143B cells was previously reported by others to inhibit colony formation, migration, and invasion ${ }^{8}$. Another microRNA, miR-199a-3p, was reported to directly target AXL to inhibit $143 \mathrm{~B}$ cell migration and invasion ${ }^{17}$. AXL has been linked with chemoresistance in a number of different cancer types ${ }^{18-20,25}$. In addition to AXL other proteins such as ATM which are also implicated in chemo-resistance ${ }^{21,26}$ were also found to be downregulated in cells treated with miR-509-3p and cisplatin which suggest that AXL downregulation could be one of the many events that lead to chemo-sensitivity in OS cells. AXL is a receptor tyrosine kinase that plays an important role in the progression of cancer by enhancing proliferation and migration, and inhibiting apoptosis and therapeutic resistance ${ }^{25}$.

AXL receptor signaling suppresses p53 in melanoma through stabilization of the MDMX-MDM2 complex and AXL inhibition is shown to increase the expression of p53 target genes and sensitize cells to cisplatin ${ }^{27}$. We found that miR-509-3p overexpression caused downregulation of AXL and upregulation of p53 (Supplementary Fig. 2). This suggests that AXL-induced modulation of the MDMX-MDM2 hetero-complex stability can suppress p53 activity, which in turn desensitizes cells to cisplatin in melanoma ${ }^{27}$. Most importantly, AXL is frequently phosphorylated (P-AXL) in OS patient samples ${ }^{8}$. Activated AXL (P-AXL) is highly expressed in OS cells, and this expression significantly correlates with the recurrence and lung metastasis of OS patients ${ }^{28}$. Furthermore, high expression of $\mathrm{P}$-AXL is predictor for worse prognosis in patients with $\mathrm{OS}^{28}$. 

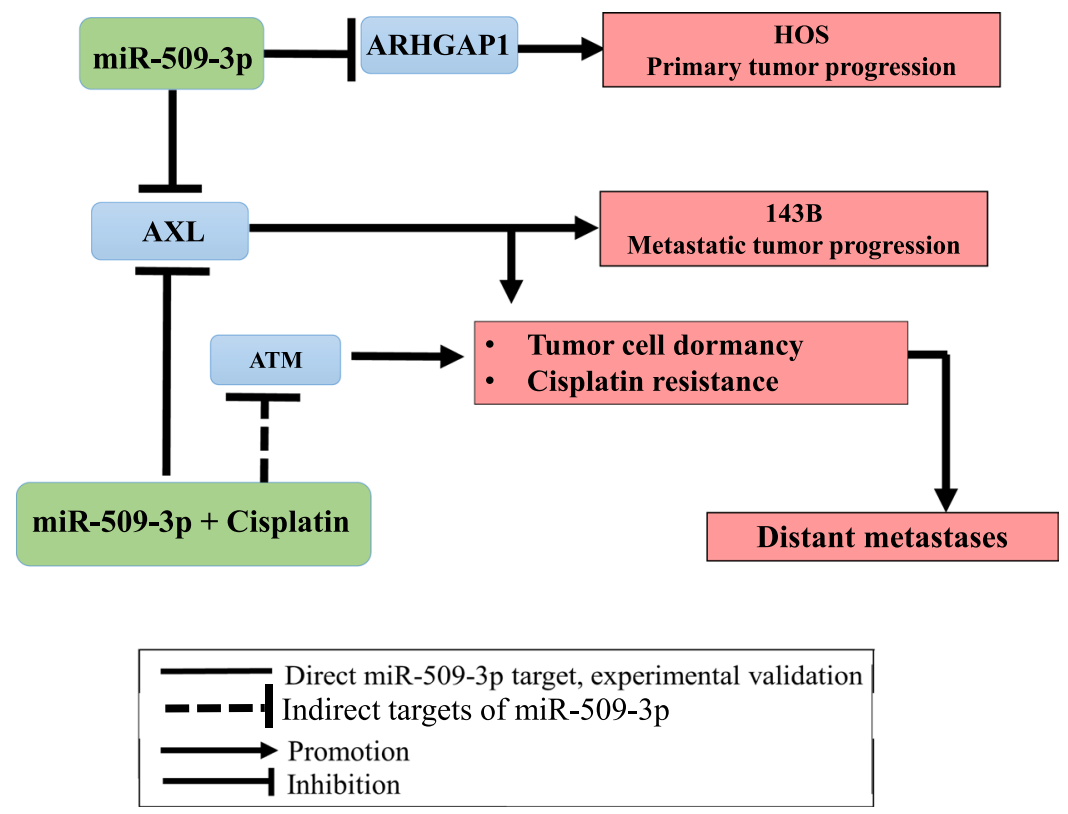

Figure 7. Model for miR-509-3p-mediated inhibition of migration/invasion and sensitization to cisplatin in OS. miR-509-3p directly inhibits ARHGAP1 in primary OS cell line HOS and AXL in metastatic OS cell line 143B to inhibit migration and invasion. miR-509-3p elicits sensitivity to cisplatin partially by directly inhibiting AXL and partially by indirectly inhibiting ATM.

As per our knowledge, this is the first report of confirming ARHGAP1 and AXL as a true direct target of miR509-3p. We have successfully demonstrated that both of these miR-509-3p targets were independently identified and validated in our osteosarcoma models and have significant biological ramifications. Further mechanisms downstream of ARHGAP1 and AXL can be explored in future which can give better understanding of role of these genes in OS metastasis and chemo-resistance. Based on GeneCards (https://www.genecards.org/), AXL and ARHGAP1 together are associated with the Actin nucleation (important for membrane trafficking, leading edge protrusion during cell migration, cell division etc.) and GPCR signaling (involved in a wide variety of physiological processes, including being involved in growth and metastasis of some types of tumors.) pathways. Based on the network from GeneMania (https://genemania.org/), AXL and ARHGAP1 both interact with PIK3 isoforms - PIK3R1 and PIK3R2 indicating a connection to the PI3K/Akt pathway. In future regulatory relations/ functions between AXL and ARHGAP1 can be studied to gain more insight into complex molecular pathways regulated by microRNAs.

Our working model for how miR-509-3p inhibits OS migration, invasion and proliferation by targeting the ARHGAP1 and sensitizes OS cells to cisplatin in part by downregulating AXL is outlined in Fig. 7. Systemic chemotherapy, which, increases long-term survival rates up to $75 \%$ in patients with localized OS has had only a minimal impact on the overall survival of patients with metastatic disease $\mathrm{e}^{5,6}$. We propose that the miR-509-3p/ AXL and miR-509-3p/ARHGAP1 axes could be used to discover new druggable targets to significantly reverse chemo-resistance and inhibit cellular migration/invasion of cell from the primary tumor, thus leading to improved clinical outcomes for patients presenting with metastatic OS.

\section{Methods}

Cell lines and growth conditions. OS cell lines HOS, 143B, U2OS, SaOS2, and LM7 were provided by Dr. Jason T. Yustein, Department of Pediatrics, Baylor College of Medicine, Houston, Texas with an MTA in place. All OS cell lines were cultured in DMEM medium (Thermo Fisher Scientific, Waltham, MA, USA) and supplemented with $10 \%$ heat-inactivated fetal bovine serum (FBS) (Atlanta Biologicals, Flowery Branch, GA, USA). All the cultures were maintained at $37^{\circ} \mathrm{C}$ in a humidified incubator with $5 \% \mathrm{CO}_{2}$.

Transient transfection/overexpression of microRNA and siRNA. Cells were seeded at 100,000 cells per well in a 6-well culture plate, or 4,000 cells per well in a 96-well culture plate (for proliferation assay), or 580,000 cells per $100 \mathrm{~mm}$ culture dish (for protein extraction). After $24 \mathrm{~h}$, cells were transfected with microRNA or siRNA by using Lipofectamine 2000 or 3000 reagent (Thermo Fisher Scientific, Waltham, MA, USA), following manufacturer's recommended protocol. Details of micorRNA and siRNA as follows; NC (cat \# 4464073); miR-509-3p (cat \# 4464067), miR-509-3p inhibitor (cat \# 4464084), siARHGAP1 (cat \# 4392420), siYAP1 (cat \# 4392420), siRAC1 (cat \# 4390824), siEGFR (cat \# AM16708) (all from Thermo Fisher Scientific, Waltham, MA, USA) and custom made siAXL (5' AGAUUUGGAGAACACACUGA 3') (Dharmacon, Lafayette, CO, USA). Three to four hours post transfection, Opti-MEM with 10\% FBS was added to each well at a volume equal to the transfection reaction volume. Twenty four hours post transfection, the transfection reaction was removed and fresh DMEM with 10\% FBS was added. 
Total RNA isolation. Total RNA was isolated at indicated time points using miRNA Easy Mini Kit (Qiagen, Venlo, Netherlands). Cells were washed twice with PBS, and harvested by centrifugation. Cell pellets were lysed using Qiazol lysis buffer (Qiagen, Venlo, Netherlands). Total RNA including the miRNA fraction was isolated following the manufacturer's instructions. The purity and concentration of the extracted RNA was determined using an ND-1000 Nanodrop spectrophotometer (Thermo Fisher Scientific, Waltham, MA, USA)

Reverse transcription and quantitative Real Time PCR (qRT-PCR/qPCR) for mRNAs. One microgram of total RNA from each sample was reverse transcribed using the high-capacity cDNA reverse transcription kit (Applied Biosystems, Foster City, CA, USA) according to the manufacturer's instructions. Reverse transcription was carried out with the Veriti Thermal Cycler system (Applied Biosystems, Foster City, CA, USA). All qPCR experiments were performed using Power SYBR Green Master Mix (Applied Biosystems, Foster City, CA, USA) on StepOnePlus Real-Time PCR System (Applied Biosystems, Foster City, CA, USA) and analyzed manually using the $\Delta \Delta \mathrm{Ct}$ method. $18 \mathrm{~S}$ or GAPDH were used as endogenous controls for all targets. All qPCR reactions were performed in three technical and at least three biological replicates.

Reverse transcription and quantitative Real Time PCR (qRT-PCR/qPCR) for microRNAs. Ten nanograms of total RNA was reverse transcribed in $15 \mu$ final reaction volume using the respective stem-loop RT primers with the TaqMan microRNA reverse transcription kit (Applied Biosystems, Foster City, CA, USA) according to the manufacturer's instructions. All qPCR experiments were performed using a Taqman Universal qPCR Master Mix without AmpErase UNG (Applied Biosystems, Foster City, CA, USA) on StepOnePlus ${ }^{\mathrm{TM}}$ Real-Time PCR System (Applied Biosystems, Foster City, CA, USA) and analyzed manually using the $\Delta \Delta \mathrm{Ct}$ method. RNU48 was used as an endogenous control. All RT-PCR reactions were performed in three technical and at least four biological replicates.

Scratch plate/wound healing assay. Scratch plate/wound healing assays were performed using the IncuCyte ZOOM Live-Cell Analysis System (Essen BioScience, Ann Arbor, MI, USA). Sixty hours post transfection, cells were collected from six well plates and seeded in 96-well ImageLock microplates (Essen BioScience, Ann Arbor, MI, USA) at a cell density of 35,000 cells per well. Cisplatin was added $(2.5 \mu \mathrm{g} / \mathrm{ml})$ at the time of seeding in appropriate wells. Twelve hours after seeding ( $72 \mathrm{~h}$ after transfection), artificial scratch wounds were made using the WoundMaker ${ }^{\mathrm{TM}}$ (Essen BioScience, Ann Arbor, MI, USA). Each well was washed twice with PBS and then overlaid with $200 \mu \mathrm{l}$ of DMEM medium supplemented with $10 \%$ FBS. Images at different time points were acquired using the IncuCyte-ZOOM ${ }^{\mathrm{TM}}$ live cell imaging system. Cell migration was calculated with IncuCyte's automated image analysis algorithm using the Relative Wound Density (RWD) metric. A single experiment included 3 technical replicates and RWD at each time point was normalized to RWD at the final time point $(40 \mathrm{~h})$ of scrambled negative RNA control (NC) to obtain relative migration. Average relative migration between independent experiments was plotted as a function of time. At least three biological replicates were used to plot relative migration. Student t-test was used for calculating statistical significance. Error bars represent standard error of mean (SEM) between biological replicates.

Luciferase reporter assay. To generate the plasmid with the wild-type target binding sequence, a region of about $500 \mathrm{bps}$, containing the predicted microRNA binding site was PCR amplified from human genomic DNA and cloned into the NotI restriction site of a psiCHECK-2 vector (Promega, Madison, WI, USA) using the Gibson Assembly Cloning Kit (New England Biolabs, Ipswich, MA, USA). For ARHGAP1, it was a 492 bp long DNA fragment ( -1438 to $-1930 \mathrm{nt})$ with the predicted miR-509-3p target site located at -1898 to $-1905 \mathrm{bp}$ in the ARHGAP1 3'-UTR. For AXL, a 501 bp long DNA fragment ( -3585 to $-4085 \mathrm{nt})$ with the predicted miR509-3p target site located at -4034 to $-4040 \mathrm{bp}$ in the AXL $3^{\prime}$-UTR was utilized. Mutant clones were generated by mutating the miR-509-3p seed interaction site in the reporter plasmid construct, using the Q5 Site-Directed Mutagenesis kit (New England Biolabs, Ipswich, MA, USA). For ARHGAP1-mut (ACCAATCA- > ACtAAgCA) and for AXL-mut (GCCAATC- > GCgAtTC). The clones were verified by Sanger sequencing. HOS cell line was plated at 10,000 cells/well in a 96-well plate. One hundred nanograms of the wild-type reporter plasmid was transfected alone (NT), or co-transfected with $20 \mathrm{nM}$ of either a scrambled negative RNA control (NC) or a miR509-3p mimic. To confirm the reduction in luciferase was due to direct binding of the microRNA to the $3^{\prime} \mathrm{UTR}$, a fourth experimental condition was included where cells were transfected with one hundred nanograms of the mutant plasmid and $20 \mathrm{nM}$ of the miR-509-3p mimic. Transfections were performed using Lipofectamine 2000 (Life Technologies, Carlsbad, CA, USA) following the manufacturer's recommended protocol. Luciferase assays were performed at $48 \mathrm{~h}$ post transfection using a Dual-Luciferase Reporter Assay System (Promega, Madison, WI, USA). Renilla luminescence signals were normalized by the firefly luciferase signals. Three biological replicates were performed for each assay.

Cell proliferation/viability assay. Cell proliferation/cell viability was assessed by MTS assay. In a 96 well plate, 4000 cells per well were seeded in DMEM media with $10 \% \mathrm{FBS}$. After $24 \mathrm{~h}$, cells were transfected with miR509-3p mimic, NC, and appropriate siRNAs. At the indicated time points ( $24 \mathrm{~h}, 48 \mathrm{~h}, 72 \mathrm{~h}$ and $96 \mathrm{~h}$ ), media from the 96-well plate was removed and $100 \mu \mathrm{l}$ pre-warmed DMEM media with $10 \%$ FBS was added. After 15-20 mins, $20 \mu$ of CellTiter $96^{\circledR}$ AQueous One Solution Cell Proliferation Assay (MTS) (Promega, Madison, WI, USA) was added to each well and the plate was incubated in the dark at $37^{\circ} \mathrm{C}$ for $1 \mathrm{~h}$ as recommended by the manufacturer. Absorbance was recorded at $490 \mathrm{~nm}$ using SpectramaxM5 plate reader (Molecular Devices, San Jose, CA, USA). Absorbance was normalized to absorbance from scrambled RNA control (SC/NC) transfected wells. Percent viability was calculated by the following formula (Absorbance of treatment well/absorbance of NC well) $\mathrm{X} 100$. Average of biological replicates was plotted with SEM. Student's $t$ test was performed to calculate statistical significance. 
Matrigel invasion assay. Cell invasion assays were performed using Matrigel-coated 96-well microplates. Plates were coated overnight with $50 \mu \mathrm{l}$ of $0.1 \mathrm{mg} / \mathrm{ml}$ Matrigel matrix (BD Biosciences, San Jose, CA, USA), before seeding 35,000 cells per well in 96 well plate cell culture plate. Scratch/wounds were generated as described for the scratch-wound migration assay. After making scratch and washing wells, $50 \mu \mathrm{l}$ of Matrigel matrix, diluted to $1 \mathrm{mg} / \mathrm{ml}$ in cell culture medium, and was added to each well. Microplates were incubated at $37^{\circ} \mathrm{C}$ to set the Matrigel and were overlaid with $200 \mu \mathrm{l}$ of culture medium $24 \mathrm{~h}$ later. Images at different time points were acquired using the IncuCyte-ZOOM ${ }^{\mathrm{TM}}$ live cell imaging system. Density was plotted as a function of time for three independent experiments, with error bars showing SEM between biological experiments. Student's t-test was used for calculating statistical significance.

Reverse phase protein array. In a six-well cell culture plate, 100,000 cell per well were seeded $24 \mathrm{~h}$ before transfection. Cells were treated with various treatments as described. Cells were harvested at $72 \mathrm{~h}$ after transfection washed twice with PBS and lysed using RPPA lysis buffer as recommended by MD Anderson Cancer Center Core Facility, Houston, TX. Cellular protein concentration was determined by Bradford assay. Protein concentration was adjusted to $1.5 \mu \mathrm{g} / \mathrm{ml}$. The cell lysate was mixed with 4 X SDS Sample Buffer with freshly added $\beta$-mercaptoethanol $(\beta-\mathrm{Me})$ as recommended by MD Anderson Cancer Center Core Facility, Houston, TX. Samples were boiled for $5 \mathrm{~min}$, and stored in $-80^{\circ} \mathrm{C}$ until sample submission. RPPA with the 295 standard antibody list used by MD Anderson Cancer Center Core Facility was performed at MD Anderson Cancer Center Core Facility, Houston, TX.

Western blots. Cells were treated as described for a period of $72 \mathrm{~h}$ and were collected and pelleted. The cell pellets were washed with PBS twice. The supernatant was discarded and the cell pellets were stored at $-80^{\circ} \mathrm{C}$ until protein extraction. Protein extraction was performed using RIPA buffer (Sigma-Aldrich, St. Louis, MO, USA) with ProBlock ${ }^{\mathrm{TM}}$ protease inhibitor cocktail (GoldBio, St Louis, MO, USA) and Simple Stop phosphatase inhibitor cocktail (GoldBio, St Louis, MO, USA). Fifty micrograms of protein per well were size separated on NuPAGE 4-12\% Bis-Tris gels using Bolt ${ }^{\mathrm{TM}}$ MOPS SDS buffer (Invitrogen, Carlsbad, CA, USA). The proteins were transferred to PVDF membranes (Invitrogen, Carlsbad, CA, USA) and blocked for an hour at room temperature with a $5 \%$ solution of non-fat dry milk dissolved in TBST $(50 \mathrm{mM}$ Tris- $\mathrm{HCl}, 150 \mathrm{mM} \mathrm{NaCl}, 0.1 \%$ Tween 20 , pH 7.5). Primary antibodies for ARHGAP1 (Abcam, Cambridge, MA, USA, cat \# ab154338), AXL (Cell Signaling Technology, Danvers, MA, USA, cat \# 8661) and GAPDH (Cell Signaling Technology, Danvers, MA, USA, cat \# 5174) were added at a concentration of 1:2000 in 5\% bovine serum albumin (Sigma-Aldrich, St. Louis, MO, USA, cat \# A9647) and incubated overnight at $4^{\circ} \mathrm{C}$. Secondary HRP-linked anti-rabbit antibodies (Cell Signaling Technology, cat \# 7074) were added at a concentration of 1:20,000 in 5\% dry milk in TBST and incubated for an hour at room temperature. Proteins were finally visualized using SuperSignal ${ }^{\mathrm{TM}}$ West Femto Maximum Sensitivity Substrate (Thermo Fisher Scientific, Waltham, MA, USA, cat \# 34095).

\section{Data availability}

All data produced during the current study are included in this article and its supplementary files.

Received: 4 September 2019; Accepted: 19 November 2019;

Published online: 13 December 2019

\section{References}

1. Kansara, M., Teng, M. W., Smyth, M. J. \& Thomas, D. M. Translational biology of osteosarcoma. Nat. Rev. Cancer 14, 722-735 (2014).

2. Botter, S. M., Neri, D. \& Fuchs, B. Recent advances in osteosarcoma. Curr. Opin. Pharmacol. 16, 15-23 (2014).

3. Rainusso, N., Wang, L. L. \& Yustein, J. T. The adolescent and young adult with cancer: State of the art - Bone tumors. Curr. Oncol. Rep. 15, 296-307 (2013)

4. Osaki, M. et al. MicroRNA-143 regulates human osteosarcoma metastasis by regulating matrix metalloprotease-13 expression. Mol. Ther. 19, 1123-1130 (2011)

5. Adamson, P. C. \& Blaney, S. M. New approaches to drug development in pediatric oncology. Cancer J. 11, 324-330 (2005).

6. Duchman, K. R., Gao, Y. \& Miller, B. J. Prognostic factors for survival in patients with high-grade osteosarcoma using the Surveillance, Epidemiology, and End Results (SEER) Program database. Cancer Epidemiol. 39, 593-599 (2015).

7. Khanna, C. et al. Toward a drug development path that targets metastatic progression in osteosarcoma. Clin. Cancer Res. 20, 4200-4209 (2014).

8. Rettew, A. N. et al. Multiple receptor tyrosine kinases promote the in vitro phenotype of metastatic human osteosarcoma cell lines. Oncogenesis 1, e34-e34 (2012).

9. Chen, X. et al. Recurrent somatic structural variations contribute to tumorigenesis in pediatric osteosarcoma. Cell Rep. 7, 104-112 (2014).

10. Bartel, D. P. MicroRNAs: Genomics, Biogenesis, Mechanism, and Function. Cell 116, 281-297 (2004).

11. Zhang, Y., Dai, Q., Zeng, F. \& Liu, H. MALAT1 Promotes the Proliferation and Metastasis of Osteosarcoma Cells By Activating the Rac1/JNK Pathway Via Targeting MiR-509. Oncol. Res. Featur. Preclin. Clin. Cancer Ther. https://doi.org/10.3727/09650401 7x14957939026111 (2017).

12. Pan, Y. et al. miR-509-3p is clinically significant and strongly attenuates cellular migration and multi-cellular spheroids in ovarian cancer. Oncotarget 7, 25930-25948 (2016).

13. Chan, L. H. et al. Hedgehog signaling induces Osteosarcoma development through Yap1 and H19 overexpression. Oncogene 33, 4857-4866 (2014).

14. Meyers, P. A. et al. Osteosarcoma: A randomized, prospective trial of the addition of ifosfamide and/or muramyl tripeptide to cisplatin, doxorubicin, and high-dose methotrexate. J. Clin. Oncol. 23, 2004-2011 (2005).

15. Ferrari, S. et al. Neoadjuvant chemotherapy with high-dose ifosfamide, high-dose methotrexate, cisplatin, and doxorubicin for patients with localized osteosarcoma of the extremity: A joint study by the italian and Scandinavian Sarcoma Groups. J. Clin. Oncol. 23, 8845-8852 (2005).

16. Karam, A. K. et al. Cisplatin and PI3kinase inhibition decrease invasion and migration of human ovarian carcinoma cells and regulate matrix-metalloproteinase expression. Cytoskeleton 67, 535-544 (2010). 
17. Tian, R. et al. miR-199a-3p negatively regulates the progression of osteosarcoma through targeting AXL. Am. J. Cancer Res. 4 , 738-73850 (2014).

18. Li, Y., Wang, X., Bi, S., Zhao, K. \& Yu, C. Inhibition of Mer and Axl receptor tyrosine kinases leads to increased apoptosis and improved chemosensitivity in human neuroblastoma. Biochem. Biophys. Res. Commun. 457, 461-466 (2015).

19. Kim, K. C., Baek, S. H. \& Lee, C. Curcumin-induced downregulation of Axl receptor tyrosine kinase inhibits cell proliferation and circumvents chemoresistance in non-small Lung cancer cells. Int. J. Oncol. 47, 2296-2303 (2015).

20. Hong, J., Peng, D. F., Chen, Z., Sehdev, V. \& Belkhiri, A. ABL regulation by AXL promotes cisplatin resistance in esophageal cancer. Cancer Res. 73, 331-340 (2013).

21. Zhang, C. et al. Inhibition of protein phosphatase 2A with the small molecule LB100 overcomes cell cycle arrest in osteosarcoma after cisplatin treatment. Cell Cycle 14, 2100-2108 (2015).

22. Kim, H., Watkinson, J., Varadan, V. \& Anastassiou, D. Multi-cancer computational analysis reveals invasion-associated variant of desmoplastic reaction involving INHBA, THBS2 and COL11A1. BMC Med. Genomics 3, https://doi.org/10.1186/1755-8794-3-51 (2010).

23. Yoon, S. et al. Inhibition of cell proliferation and migration by miR-509-3p that targets CDK2, Rac1, and PIK3C2A. Mol. Cells 37, 314-321 (2014).

24. Ahn, Y. H. et al. ZEB1 drives prometastatic actin cytoskeletal remodeling by downregulating miR-34a expression. J. Clin. Invest. 122, 3170-3183 (2012).

25. Axelrod, H. \& Pienta, K. J. Axl as a mediator of cellular growth and survival. Oncotarget 5, 8818-8852 (2014).

26. Engert, F., Kovac, M., Baumhoer, D., Nathrath, M. \& Fulda, S. Osteosarcoma cells with genetic signatures of BRCAness are susceptible to the PARP inhibitor talazoparib alone or in combination with chemotherapeutics. Oncotarget 8, 48794-48806 (2017).

27. De Polo, A. et al. AXL receptor signalling suppresses p53 in melanoma through stabilization of the MDMX-MDM2 complex. J. Mol. Cell Biol. 9, 154-165 (2017).

28. Han, J. et al. Gas6/Axl mediates tumor cell apoptosis, migration and invasion and predicts the clinical outcome of osteosarcoma patients. Biochem. Biophys. Res. Commun. 435, 493-500 (2013).

\section{Acknowledgements}

This work was made possible through generous funding from a number of sources including; Moores Chair (P.H.G.), Robert \& Janice McNair Foundation (S.L.P.\& A.P.), McCammon Foundation (A.P.), NIH P30 shared resource grant CA125123, NIEHS P30 Center grant 1P30ES030285, and CPRIT grant RP170005 (K.R. \& C.C.). We would like to thank Dr. Sujash Chaterjee for critically reading the manuscript.

\section{Author contributions}

S.L.P. designed and performed; migration, proliferation, invasion, RPPA, siRNAs based gene knockdown experiments; analyzed data, and drafted and wrote manuscript. A.P. performed western blots and luciferase reporter assays, Y.P. edited manuscript, K.R. and C.C. analyzed RPPA data and edited manuscript, M.B., R.M. and J.T.Y. revised the manuscript critically for important and intellectual content. J.T.Y. provided cell lines, P.H.G. drafted and revised the manuscript critically for important and intellectual content. All authors read and approved the final manuscript.

\section{Competing interests}

Authors have no competing interests as defined by Nature Research, or other interests that might be perceived to influence the results and/or discussion reported in this paper.

\section{Additional information}

Supplementary information is available for this paper at https://doi.org/10.1038/s41598-019-55170-2.

Correspondence and requests for materials should be addressed to P.H.G.

Reprints and permissions information is available at www.nature.com/reprints.

Publisher's note Springer Nature remains neutral with regard to jurisdictional claims in published maps and institutional affiliations.

(c) Open Access This article is licensed under a Creative Commons Attribution 4.0 International

License, which permits use, sharing, adaptation, distribution and reproduction in any medium or format, as long as you give appropriate credit to the original author(s) and the source, provide a link to the Creative Commons license, and indicate if changes were made. The images or other third party material in this article are included in the article's Creative Commons license, unless indicated otherwise in a credit line to the material. If material is not included in the article's Creative Commons license and your intended use is not permitted by statutory regulation or exceeds the permitted use, you will need to obtain permission directly from the copyright holder. To view a copy of this license, visit http://creativecommons.org/licenses/by/4.0/.

(c) The Author(s) 2019 